\title{
L'elettrocardiografia endocavitaria per la valutazione della posizione della punta dei cateteri venosi centrali
}

\author{
F. Ariel Sánchez ${ }^{1}$, R. Picca Nicolino ${ }^{1}$, F. Fiorini ${ }^{2}$ \\ ${ }^{1}$ U.O. Cardiologia, Sanremo, ASL 1 Imperiese \\ ${ }^{2}$ U.O. Nefrologia e Dialisi, Sanremo, ASL 1 Imperiese
}

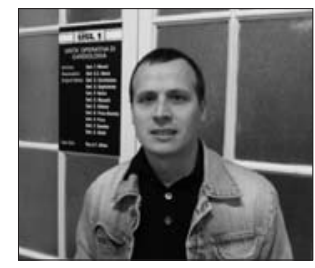

U a cateterizzazione dei vasi venosi centrali è una pratica assai frequente nella F. Ariel Sánchez cura dei pazienti critici. Circa sei milioni di impianti di cateteri venosi centrali (CVC) sono eseguiti ogni anno negli USA e circa 200000 per anno in UK (1).

Il posizionamento del CVC può essere associato a potenziali complicanze fatali (traumatiche, trombotiche e/o infettive). I primi due tipi sono intimamente relazionate a un inadeguato posizionamento della punta (tip) del catetere. Il punto anatomico di minor rischio per il posizionamento del tip del CVC lo troviamo a livello della crista terminalis (elemento anatomico che corrisponde esattamente alla giunzione tra la vena cava superiore (VCS) e l'atrio destro) (2). Shuster et al (3) suggeriscono addirittura di posizionare il tip del CVC qualche centimetro prima della crista terminalis per ridurre ancora di più il rischio di tamponamento cardiaco, poiché il pericardio viscerale tende a proiettarsi cefalicamente, allontanandosi dal cuore, coinvolgendo la VCS per circa 3-5 centrimetri (4).

Il metodo più comunemente usato per il controllo della posizione del CVC è la radiografia standard del torace (raramente la fluoroscopia). Una ulteriore possibilità di controllo del tip del CVC è permesso dall' elettrocardiografia endocavitaria ECG-EC. I primi studi di elettrofisiologia endocavitaria mediante l'uso del CVC come elettrodo intracavitario risalgono al 1949 (5).

Serafini et al (6-8), negli anni Ottanta, hanno descritto la tecnica per il posizionamento della punta dei CVCs tramite elettrocardiografia endocavitaria (ECG-EC), che per la sua semplicità, sicurezza ed efficacia in confronto alle altre metodiche è divenuta attualmente molto diffusa: d'altra parte si tratta di una tecnica con un rapporto costo-beneficio più favorevole $(5,9-12)$. In alcuni ospedali ha proprio sostituito il controllo radiologico post-impianto dei CVC nei reparti di rianimazione. Anche in sede extraospedaliera (ad esempio, ambulanza) il controllo con ECG-EC per l'impianto dei CVC ha dimostrato di essere una metodica sicura, fattibile, capace di ridurre in modo significativo il numero di malposizionamenti del catetere (13).

\section{Fenomeni elettrofisiologici}

Certi tessuti specializzati come ad esempio il muscolo o le cellule neuronali, per poter svolgere le proprie attività utilizzano l' energia elettrica endocellulare. Questa energia elettrica è presente sotto forma di differenza di potenziale (DP) elettrico transmembrana, cioè la DP relativa esistente tra lo spazio intracelullare ed extracellulare, dovuta alle differenti concentrazioni ioniche presenti tra i due spazi biologici. Questa DP transmembrana viene creata dalla diversa permeabilità della membrana plasmatica agli ioni e viene mantenuta principalmente attraverso l'attività della pompa $\mathrm{Na}+\mathrm{K}+$ ATPasi dipendente con consumo energetico sotto forma di adenosina trifosfato (ATP), molecola ad alta energia che come sappiamo è il prodotto finale della respirazione cellulare.

Le cellule muscolari cardiache hanno una DP a riposo da $-80 \mathrm{mV}$ a $-90 \mathrm{mV}$ : queste DP transmembrana sono misu- 


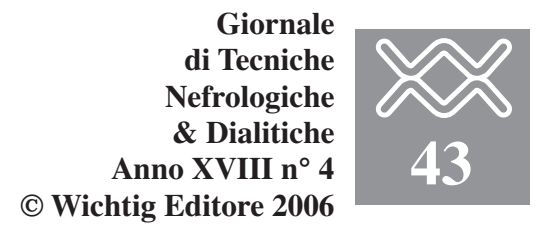

rabili in laboratorio tramite il galvanometro, posizionando un microelettrodo $(0.5 \mu \mathrm{m})$ in posizione intracellulare e un altro nello spazio extracellulare. D'altra parte è importante ricordare che a livello della superficie cardiaca, cioè in due punti diversi della superficie atriale o di quella ventricolare, esistono differenze di potenziale. Infatti se prendiamo in considerazione due punti diversi della superficie atriale e misuriamo la differenza di potenziale esistente tra i due punti tramite il posizionamento di due elettrodi, avremo una DP superficiale che varia a seconda del momento del ciclo cardiaco in cui ci troveremo. Sono proprio quest'ultime DP superficiali che vengono registrate sull'elettrocardiogramma di superficie a 12 derivazioni. Lo schema della Figura 1 rappresenta sia quello che succede in una singola cellula miocardica che nell'intero muscolo cardiaco.

Il miocardio è un tessuto eccitabile, ciò vuol dire che uno stimolo adeguato della membrana cellulare produce un cambio nella DP transmembrana (da $-90 \mathrm{mV}$ a $+20 \mathrm{mV})$ : questa DP viene chiamata potenziale d'azione (Pd'A) e positivizza l'intracellulare. Anche il miocardio, come il tessuto nervoso, ha la proprietà di condurre il $\mathrm{Pd}$ 'A lungo la stessa cellula e alle cellule contigue depolarizzando tutto il tessuto eccitabile.

Dopo la depolarizzazione del tessuto eccitabile (Fig. 1B), l'intracellulare cambia polarità diventando più positivo dell'extracellulare. Nella ripolarizzazione la cellula, tramite l'attivazione di diversi canali ionici specifici e al funzionamento della pompa $\mathrm{Na}+\mathrm{K}+\mathrm{AT}-$ Pasi dipendente, torna allo stato di riposo, diventando nuovamente eccitabile.

Nel miocardio normale lo stimolo elettrico cardiaco nasce a livello del nodo del seno [Pace Maker (PM) naturale] che provvede con ritmo regolare a stimolare le fibre miocardiche contigue dell'atrio destro. Esso genera un Pd'A che depolarizza in sequenza tutte e due gli atri e passa quindi alla giunzione AV e ai ventricoli: questi tre passaggi saranno rappresentati in successione sull' ECG come onda P, intervallo PQ e complesso QRS.

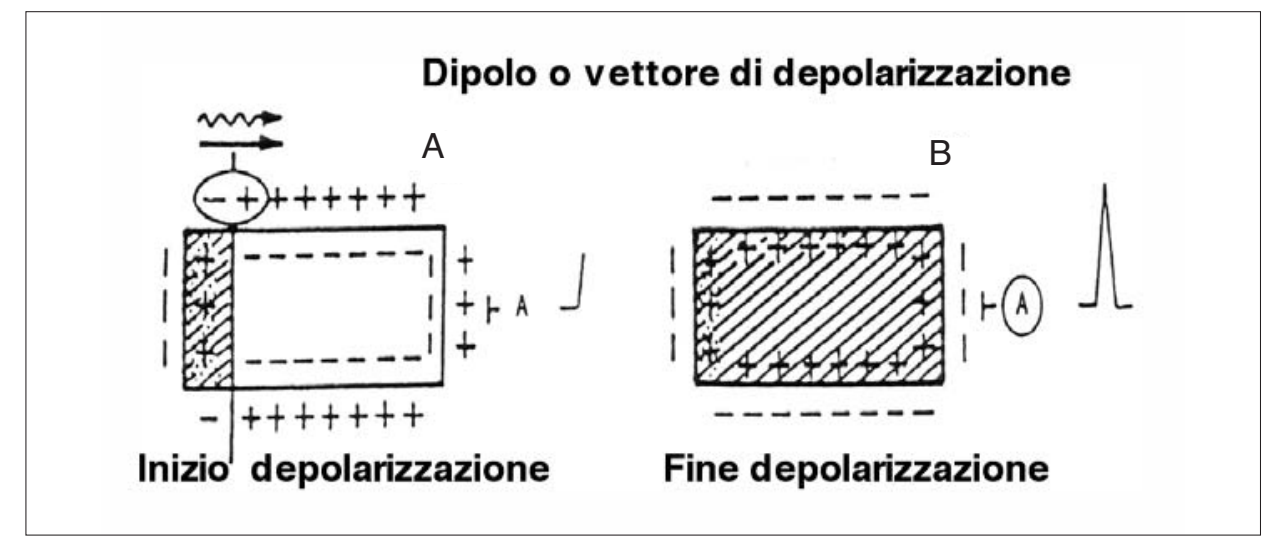

Fig. 1 - A) Il dipolo di depolarizzazione si avvicina all' elettrodo "A" il quale registra una deflezione positiva. B) Finita la depolarizzazione, sulla superficie miocardica rimangono solo cariche negative (cioè scompaiono le DP di superficie): quindi dopo la deflezione positiva il registro torna alla linea di base.

Ad esempio, se l'elettrodo "A" si trovasse sul lato opposto il suo registro diventerebbe negativo, perché "vedrebbe" la coda negativa del dipolo. Questo fenomeno è alla base della formazione e della polarità delle onde sull'ECG di superficie.

\section{L'interpretazione dell'ECG-EC: analisi vettocardiografica dell'attività elettrica atriale}

Dalla considerazione che la posizione ritenuta ottimale per il tip dei CVC sia la zona compresa fra la parte bassa-terminale della vena cava superiore e il primo tratto-iniziale dell'atrio destro, prende avvio l'idea di utilizzare il CVC come un elettrodo ECG endocavitario al fine di capire, tramite la valutazione delle differenti morfologie assunte dall'onda $\mathrm{P}$, l'esatto punto in cui il tip si trova.

La depolarizzazione degli atri genera delle differenze di potenziali (DP) a livello della superficie del muscolo atriale (Fig. 1), che cambiano progressivamente di voltaggio durante la sistole fino a scomparire completamente quando il miocardio atriale si è completamente depolarizzato (Fig. 1B). Le DP a livello delle pareti atriali si possono analizzare matematicamente e descrivere graficamente tramite l'analisi dei vettori, in base al concetto di vettore elettrico o dipolo (Fig. 1). Questi innumerevoli vettori istantanei hanno un orientamento nei tre piani anatomici determinati dalla polarità positiva del vettore (PF: piano frontale; PS: piano sagittale; PC: piano craniale) e hanno una ampiezza in relazione al voltaggio della DP. Questi infiniti vettori originano in uno stesso punto (il nodo del seno) (Fig. 2): i vettori iniziali rappresentano la depolarizzazione della parete mediale dell'atrio destro, i vettori medi rappresentano la depolarizzazione della parete laterale dell'atrio destro e mediale dell'atrio sinistro, mentre i vettori finali sono prodotti dalla depolarizzazione finale dell'atrio sinistro rimanente. Questa sequenza viene raffigurata nella Figura 2 con i tre vettori principali numerati progressivamente dal $1^{\circ}$ al $3^{\circ}$.

La depolarizzazione atriale descritta genera a livello dell'elettrocardiogramma di superficie l'onda $\mathrm{P}$ che viene seguita dall'intervallo PQ e dopo dal complesso QRS.

A questo punto risulta comprensibile, perché sull'ECG di superficie dei pazienti con ritmo sinusale l'onda $\mathrm{P}$ è negativa in aVR e positiva in DII.

Nella Figura 2 il tracciato di superficie appartenente alla derivazione frontale aVR (aVR registra o "guarda" le DP presenti sulla superficie del cuore dalla spalla destra), quindi "vedrà" tutte e tre i vettori principali dell'attività atriale allontanarsi e di conseguenza registrerà sul tracciato un'onda P negativa. Il fenomeno opposto si verifica con la derivazione DII che "guarda il cuore" dal basso e da sinistra, dando luogo a un'onda P positiva sull'ECG di superficie.

Le onde P sull'ECG di superficie sono inoltre di minore voltaggio (fino a 


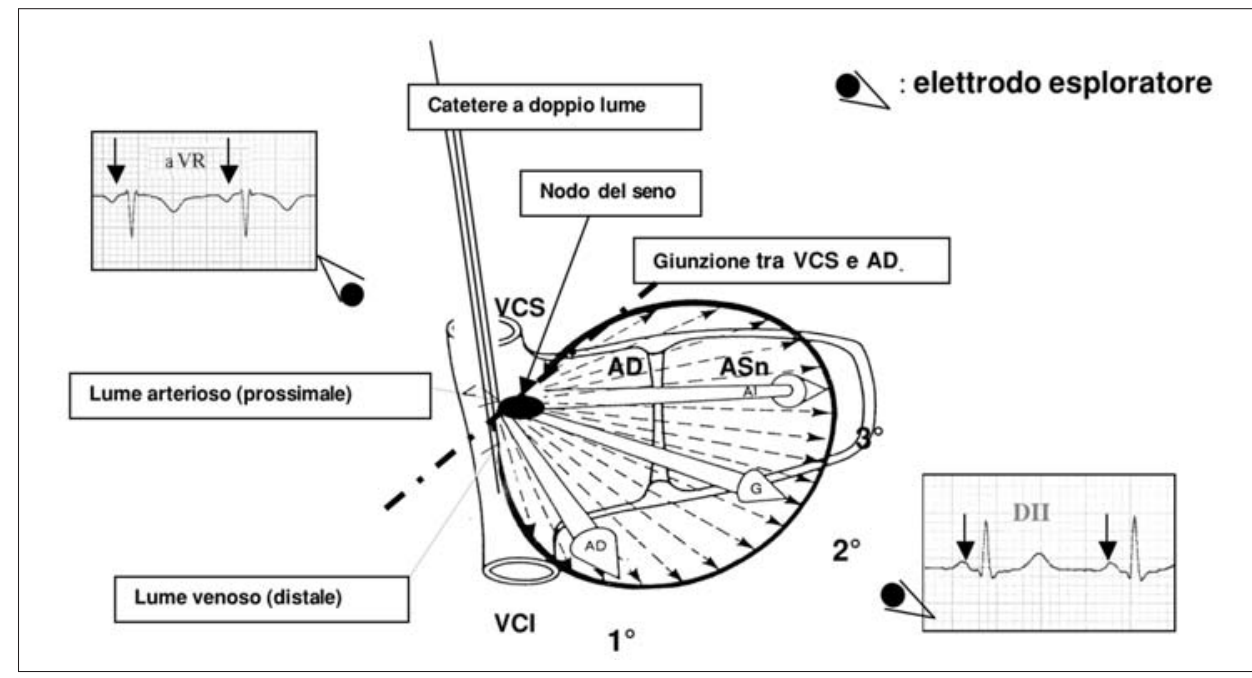

Fig. 2 - CVC a doppio lume impiantato adeguatamente. Rapporti anatomici e fenomeni vettorcardiografici utili per l'analisi della formazione dell'onda P a livello dell'ECG di superficie (vedasi le frecce sui tracciati; le onde P in AVR: negative e in DII: positive).

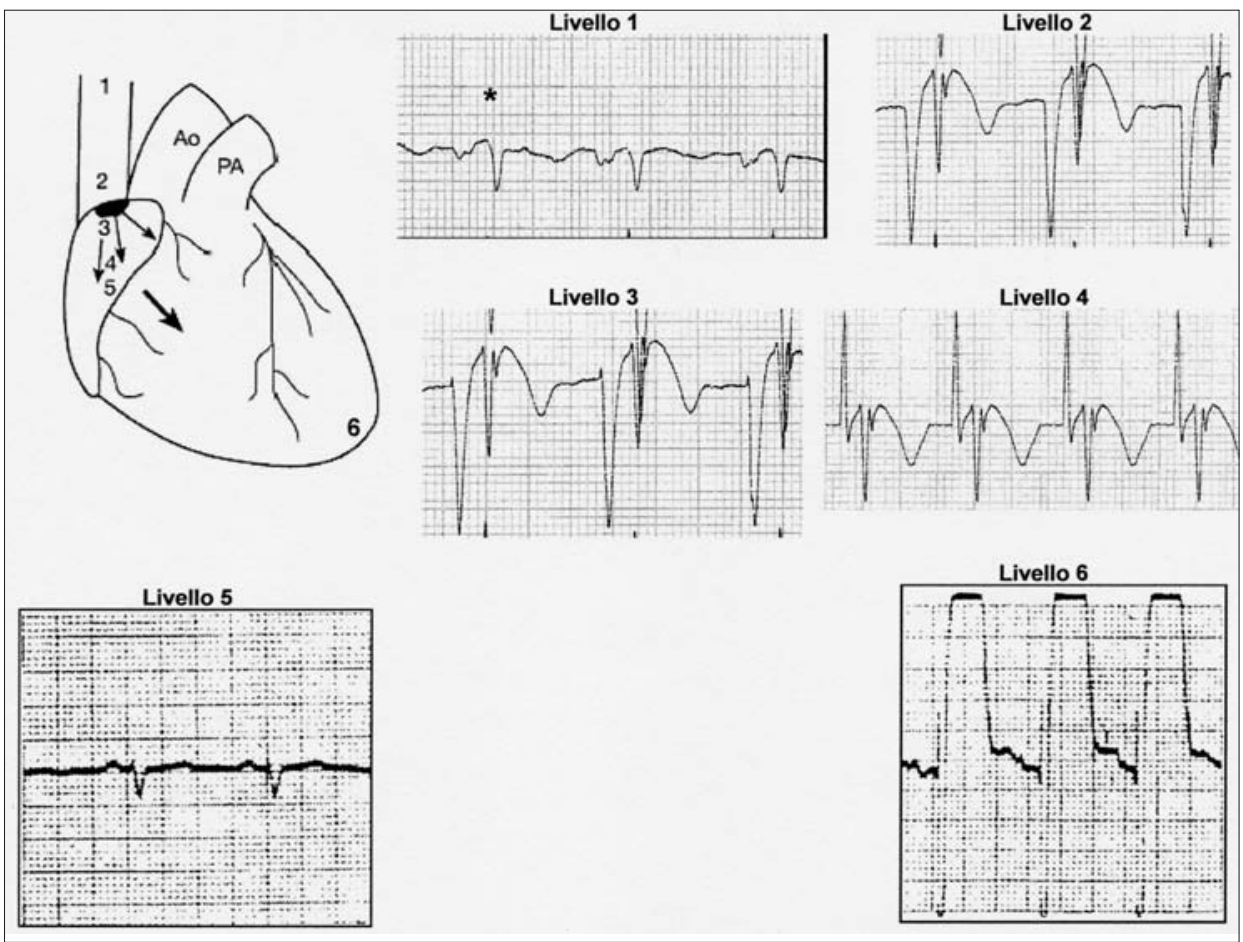

Fig. 3 - Spiegazione nel testo. Le registrazioni ECG-EC sono state eseguite dagli Autori presso l'UTIC dell'Ospedale di Sanremo.

$0.25 \mathrm{mV}$ ) rispetto al complesso QRS (tra $1 \mathrm{e} 4 \mathrm{mV}$ ): questo fenomeno ovviamente è dovuto a una minore massa muscolare atriale (in confronto a quella ventricolare) che determina DP di minore ampiezza.

\section{Registrazione dell'ECG-EC dalla vena cava superiore all'atrio destro}

La Figura 3 mostra una veduta frontale del cuore con in primo piano gli ele- menti anatomici (VCS e atrio destro) che si rapportano con il CVC nel momento del posizionamento: si possono osservare dei punti di riferimento (livelli), numerati da 1 a 6 , posti su punti anatomici diversi, da dove sono stati registrati (con un elettrodo esploratore) degli ECG-EC che vengono mostrati sulla parte destra della Figura.

Se con un elettrodo-EC posto in VCS prossimale (livello 1) si registra un tracciato ECG, si rileveranno i vettori dell'attività elettrica atriale sempre in allontanamento; quindi, avvicinandosi progressivamente alla crista terminalis, le onde $\mathrm{P}$ acquisteranno una maggiore ampiezza, ma sempre di polarità negativa (livello 2). Se l'elettrodo intracavitario oltrepassa di poco in senso caudale il nodo del seno (atrio destro alto), registrerà inizialmente i pochi vettori che si avvicinano sotto forma di una piccola deflessione positiva e tutti i restanti, che si allontano, come una deflessione negativa più grande (livello 3 ), definendo in tal modo il pattern ECG-EC dell'atrio destro alto. Mentre ci addentriamo nell'atrio destro, la deflessione positiva dell'onda P aumenta (livello 4). Se poi si posiziona l'elettrodo lontano dall'atrio destro oppure nella VCI (livello 5), l'ampiezza dell'onda $\mathrm{P}$ sarà di minore voltaggio e totalmente positiva poiché tutti i vettori della depolarizzazione degli atri sono sempre orientati in basso. Se ora l'elettrodo esploratore è posizionato a livello intraventricolare destro, in contatto con l'endocardio, l'ECG-EC registra un'onda di lesione subendocardica rappresentata da un sopraslivellamento del tratto ST (livello 6).

In emodialisi vengono utilizzati di solito cateteri a due vie (o doppi), che presentano un orifizio in punta e un altro circa 1-3 centimetri sopra. Nella posizione definitiva il tip dovrà rimanere leggermente sopra la crista terminalis, cioè nella porzione più distale della VCS oppure leggermente sotto la crista terminalis a condizione che l'orifizio prossimale sia posizionato sempre in VCS (vedi livello 2).

Il CVC deve essere riempito di soluzione salina oppure di soluzione di bicarbonato di Na che per le loro ottime proprietà conduttrici costituiscono una colonna di acqua elettricamente attiva in 


$\begin{array}{r}\text { Giornale } \\ \text { di Tecniche } \\ \text { Nefrologiche } \\ \text { \& Dialitiche }\end{array}$
$\begin{array}{r}\text { Anno XVIII n } \\ \text { 4 }\end{array}$
(c) Wichtig Editore 2006

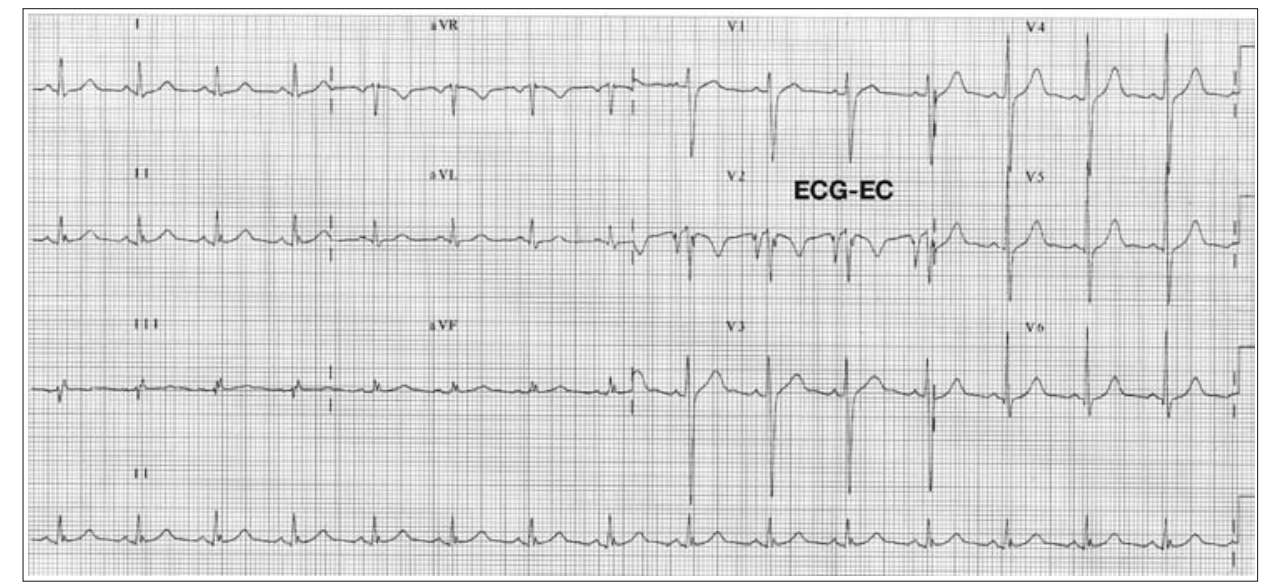

Fig. 4 - ECG a 12 derivazioni con V2 come derivazione endocavitaria "ECG-EC”.

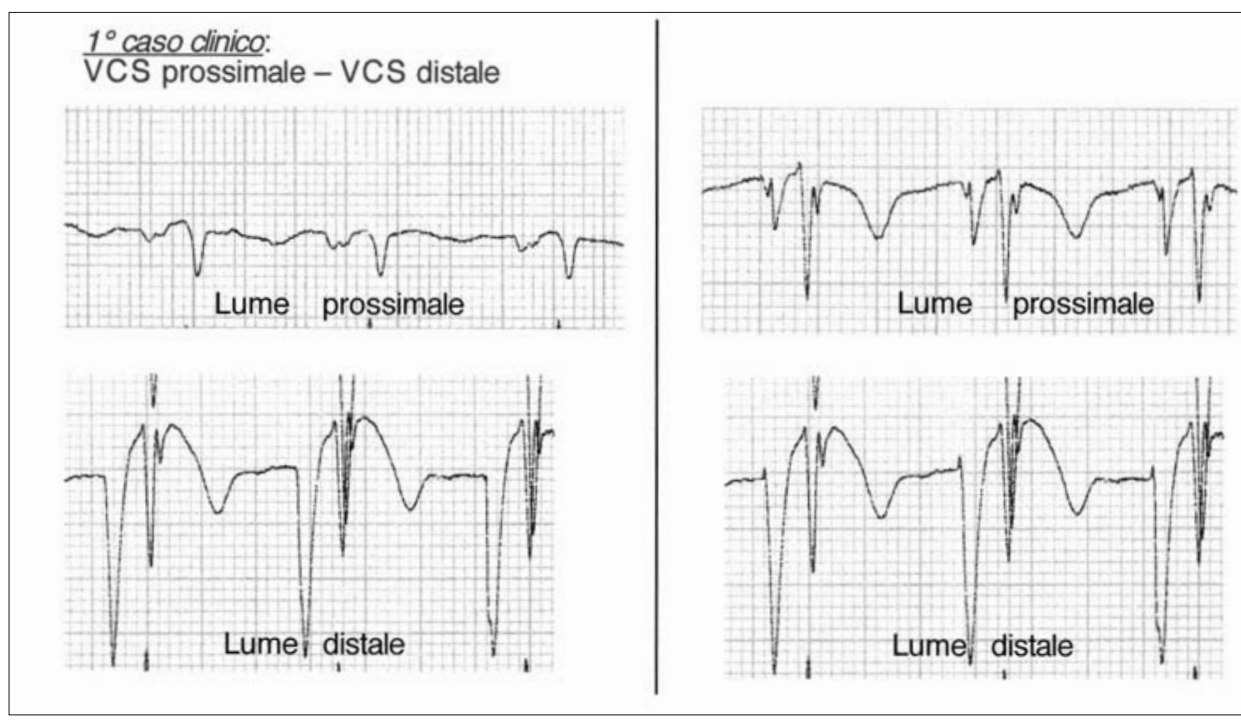

Fig. 5A - Posizionamento adeguato. Vedere spiegazione nul testo.

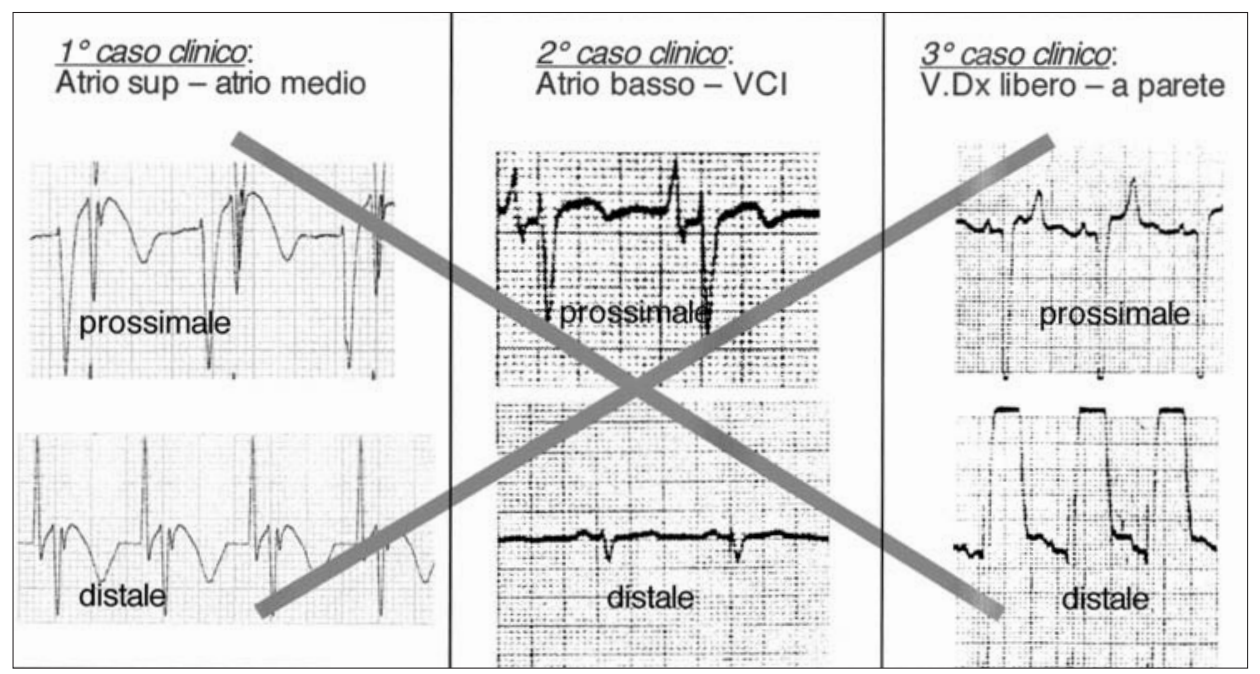

Fig. 5B - Posizionamento non adeguato. grado di rilevare l'attività elettrica endocavitaria, funzionando come un elettrodo esploratore. Il livello d'esplorazione sarà sulla punta del catetere, se usiamo il lume venoso oppure alcuni centimetri prima, se viene usato il lume prossimale o arterioso. Il concetto di usare il catetere stesso come elettrodo esploratore, o in altre parole come derivazione endocavitaria, è il fondamento dell'utilizzo ECG-EC per il corretto posizionamento del tip del CVC, permettendo, tramite le registrazioni ECGEC da entrambi i lumi, di seguire "passo per passo" la posizione del catetere in corso di impianto.

Alcuni cateteri vengono già dotati dalla casa produttrice di guide metalliche che si introducono nei lumi per eseguire le registriazioni ECG-EC: l'uso di queste anime metalliche è efficace, ma possono aumentare il rischio di complicazioni (14). L'uso di soluzioni saline come conduttori elettrici è altrettanto efficace e più sicuro, quindi è consigliabile quest'ultima modalità.

\section{Materiale necessario}

1) Un catetere centrale a doppio lume dotato di tappi emostatici perforabili ("injection cap").

2) Due siringhe da $10-20 \mathrm{ml}$, piene di soluzione salina, provviste del proprio ago.

3) Una flebo da $100 \mathrm{~mL}$ di soluzione fisiologica.

4) Cavo elettrico sterile dotato di due pinzette "a coccodrillo" alle estremità.

5) Un elettrocardiografo.

\section{Procedura}

- Eseguire un ECG di superficie di 12 derivazioni per verificare che il paziente sia in ritmo sinusale; in assenza di tale conferma è consigliabile usare un altro metodo di controllo per la valutazione del posizionamento del tip del CVC.

- Posizionare il CVC con ecoguida in vena giugulare interna.

- Lavare entrambi i lumi con fisiologica per eliminare eventuali bolle d'aria e posizionare gli "injection cap". 
- La siringa da $10 \mathrm{ml}$ o $20 \mathrm{ml}$ carica di soluzione bicarbonato di $\mathrm{Na}+$ con il suo ago collegato.

- Pungere con le due siringhe piene di fisiologica i due tappi emostatici perforabili e far defluire $2-3 \mathrm{~mL}$ di soluzione fisiologica in ogni lume.

- Connettere con il cavo elettrico a pinzette l'ago metallico con la derivazioneV2 dell'elettrocardiografo (utilizzare eventualmente gel sterile).

- Procedere alla registrazione ECG endocavitaria iniziando dal lume distale.

- Collegare tutte le altre derivazioni al Paziente ed eseguire un ECG di superficie a 12 derivazione di cui V2 sarà la derivazione endocavitaria $(\mathrm{EC})$ (Fig. 4).

Se la punta del catetere si trova in VCS prossimale nella derivazione V2 si evidenzierà un'onda $\mathrm{P}$ negativa di basso voltaggio, oppure se il tip si trova alla giunzione con l'atrio destro la $\mathrm{P}$ sarà negativa e di voltaggio più che raddoppiato; spingendo delicatamente il catetere alcuni millimetri in avanti, l'onda $\mathrm{P}$ nella derivazione $\mathrm{V} 2$ diventerà positiva e negativa (pattern atrio destro alto o medio), oppure diventerà totalmente positiva se in atrio basso. Nella Figura 5 sono mostrati gli ECG di 5 pazienti diversi eseguiti tramite ECG-EC: ogni paziente ha eseguito la registrazione endocavitaria sia dal lume distale o venoso che dal prossimale o arterioso.

\section{BIBLIOGRAFIA}

1.

Schummer W, Herrmann S, Shummer C, Funke F, et al. Intraatrial ECG is not a reliable method for positioning left internal jugular vein catheters. Br J Anaesth 2003; 91 (4): 481-6.

2.

Fletcher S J and Bodenham AR. Editorial II. Safe placement of central venous catheters: Where should the tip of the catheter lie? Br J Anaesth 2000; 85 (2):188-9.

3. Schuster M, Nave H, Piepenbrock $\mathrm{S}$, et al. The carina as landmark in central venous catheter placement. Br J Anaesh 2000; 85: 192-4.

4. Shummer W, Schummer C, Muller A, et al. ECG-guided central venous catheter positioning: does it detect the pericardial reflection rather than the right atrium. Eur J Anaesth 2004; 21 (8): 600-5.

5. Hellerstein HK, Pritchard WH, Lewis RL. Recording of intracavity potentials through a single lumen, saline filled cardiac catheter. Proc Soc Exper Biol \& Med 1949; 71: 58 .

6.

Galli F, Efficace E, Villa G, Salvadeo A, Criffo A Paroni G, Serafini G. Endocavitary eletrocardiography /EC-ECG in monitoring central venous cannulation for vascular access in haemodialysis. Nephrol Dial Transplant 1993; 8(5): 480-1.

7. Serafini G, Petrobono, Parigi GB, Cornara G. Positioning of a central venous catheter in children by intracavitary ECG. A new technic. Minerva Anestesiol 1985; 51(6): 289-91.

8.

Serafini G, Petrobono, Cornara G. Location of central venous catheters in children by endocavitary ECG: A new technique. Clin Nutr 1985; 4(4): 201-2.

9. Tierney SN, Katke J, Langer JC. Cost Comparison of Electrocardiography Versus Fluoroscopy for Central Venous Line Positioning in Children. J Am Coll Surg 2000; 191: 209-11.

10. Cheng KI, Chu Ks, Chen LT,
Tang CS. Correct positioning of venous port-a-cath catheter: comparisonof intravascular electrocardiography signal from guidewire and sodium bicarbonate flushed catheter. Anaesth Intensive Care 2002; 30(5): 603-7.

11. Cheng KI, Chu Ks, Yu Kl, Lu V, Chen HM, Tang CS. A novel approach of intravenous electrocardiograph technique in correct position the longterm central venous catheter. Kaohsiung J Med Sci 2000; 16(5): 241-7.

12. Chu KS, Hsu JH, Wang SS, Tang CS, Cheng KI, Wang CK, Wu JR. Accurate central venous por-A catheter placement: intravenous electrocardiography and surface landmark techniques compared by using transesophageal echocardiography. Anaesth Anal 2004; 98(4): 910-4.

13. David JS, Tazaroute K, Perfus, Savary D. Is ECG-guidance a helpful method to correctly position a central venous catheter during prehospital emergency care? Abstract. Acta Anaesthesiol Scand 2005; 49; 1010.

14. Dionisio P, Valenti M, Cornella C, Caramello E et al. Monitoriong of central venous dual-lumen catheter placement in haemodialysis: improvement of a technique for the practising nephrologist. Nephrol Dial Transplant 1995; 10: 874-6. 